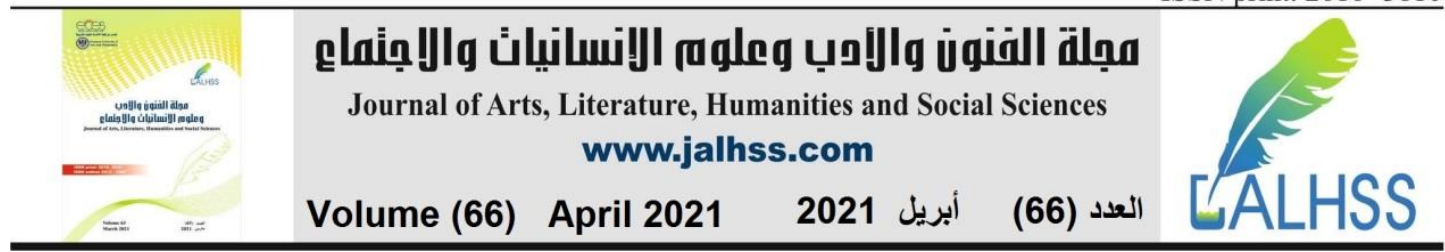

\title{
The Oppressed Disability Sees The Music
}

\author{
Hadda Zoghbi \\ Algerian resident in the Kingdom of Bahrain \\ Email: hadda.zoghbi@gmail.com \\ Dr. Karim Ben Messai \\ Department of Physics, College of Science, University of Bahrain, Kingdom of Bahrain \\ Email: kmessai@uob.edu.bh
}

\begin{abstract}
In the perspective of finding new ways of helping the deaf community, specifically in helping them understand music, this article introduces a new method of linking sounds to colors according to HK scale (abbreviation: Hadda and Karim). This scale is based on a direct calculation method shifting the frequency of an audible musical note to reach the frequency of a known-colored light. The physical medium in which the sound wave $(10 \mathrm{~Hz}-20 \mathrm{kHz})$ propagates, such as air or steel, is taken into consideration to calculate its high order harmonic in the visible light range (400700nm). HK scale associates to each note of music a color. This new method allows the creation of beautiful drawings as interpretation of musical pieces in a scientific way not based only on Synaesthesia. Some artworks of what music looks like are presented in the article, Beethoven sonata No. 14 (piano) for the listener and for the deaf, and the Bahraini National Anthem for the listener and for the deaf. This technique could be used to communicate with deaf people. It shows how important Art could be used in our daily life in different area. It means that Art is life.
\end{abstract}

Keywords: Deafness, Art, Music, Color, Psychology. 


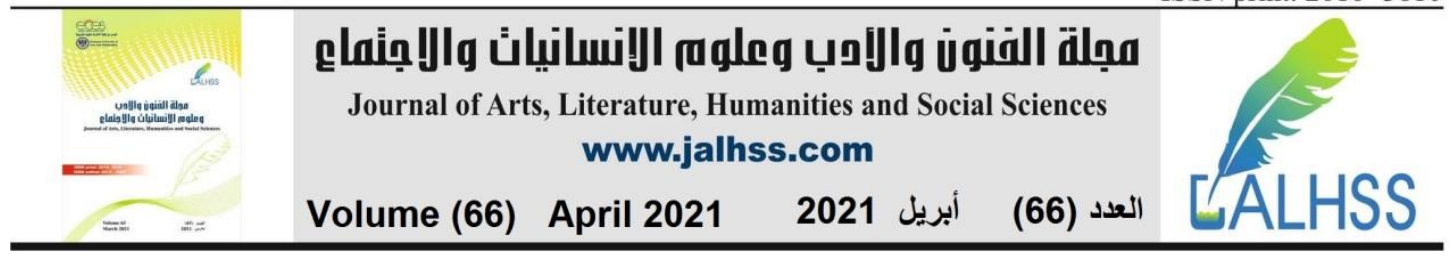

\section{Introduction}

Globally, 466 million people suffer from disabling hearing loss, and 34 million of them are children. It is estimated that by 2050 , more than 900 million people, or one in ten people, will suffer from a hearing loss (WHO 2020). What is more painful is that society describes deaf people as tending to isolation and introversion and confining themselves in their own community. The main reason for that may be due to the weakness of communication between them and the normal people, which may not find the need to learn sign language. Fortunately, this introversion that may generate mental illnesses and complexes can be treated with arts. Plastic art is distinguished from others by being an effective means of expressing the underlying feelings and an effective psychological and rehabilitation treatment for many chronic and incurable diseases in society, specifically for this type of group, the deaf group or as it is sometimes called oppressed disability (Mohamad 2011).

Among the shortcomings experienced by this group is their inability to enjoy the beauty of music. As Aldous Huxley says (Aldous 2014): "After silence, that which comes nearest to expressing the inexpressible is music." Although deaf people know silence, they fail to approve of music, and this impotence prevents them from enjoying silence.

Here it is worth mentioning the life story of the famous musician, Beethoven, and his suffering at the end of his life, a disease of gradual loss of the sense of hearing, and how it reached a stage in which he became deaf (Greenberg 2001). Despite that he produced his best music while he did not hear. How can a deaf person compose a successful piece of music when he did not hear it?! Not to mention the deaf who never heard music in his life or stopped listening to it for a long time?!

Based on these points, this article tries to present a new method in plastic art that links sound and color as a solution that allows the deaf to appreciate music through sight. Any graphic drawing of a piece of music, or further, playing a piece of music for a visual board could be possible.

Many people may ask about the possibility of hearing a color or seeing a sound?

\section{Synaesthesia}

From a historical perspective, the idea of musical association with the visual arts may be traced back to ancient Greece, and it is mentioned that Plato was the first to talk about rhythms and music and their relationship to color (Bredlow 2010).

Also, it remains unclear whether Beethoven, who called B minor "black key", and D major "orange key", Scrippen linked E\# major to reddish-violet, Rimsky-Kurzakoff 


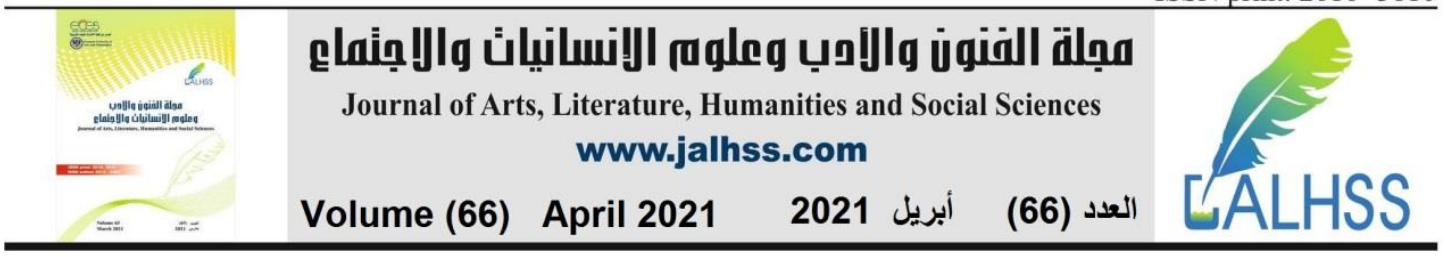

described D major in golden brown, and Schubert who saw E minor as "a maiden in a white dress with a pinkish-red bow on her chest", facts or mere illusions (Latest News 2013).

We also do not forget the famous painter Vasily Kandinsky (Ward 2014), who is credited with drawing the first abstract paintings in the history of art. It does not excite only the eye but also the ear. It is believed that Kandinsky was going through a phenomenon that psychologists call "Synaesthesia", a Greek word referring to a condition that allows a person to appreciate sounds, colors, and words with two or more senses at the same time. But Kandinsky's style may raise questions about his scientific knowledge of color and sound, as his paintings refer to a scientific method that links music to certain geometric shapes that may be related to the timing and volume of the note (mezzo-piano, mezzo-forte, forte, ...). In his book, he described in detail the colors and the link between them, but he associated colors to instruments rather than notes (Kandinsky 1911).

The first reliable case of the phenomenon of simultaneous sensation is attributed to the English philosopher and thinker John Locke, who in 1690 spoke of a man who claimed to see a crimson color whenever he heard a trumpet playing. But it remains unclear whether the composers reported something of a syncopated state or were only influenced by what Goethe, Schopenhauer and Rudolf Steiner wrote about color theories and the mixing of the senses (Ward 2014). The skeptics rejected the synesthesia as nothing more than a self-invention, such as a bad case of sensing (Lush 2020). At the end, anyone can claim that he feels and feels this sound is red and that sound is green, and another claims that he feels and feels the opposite, and this appears in the research conducted by linguists from Radbud University and the University of Edinburgh discussed the similarities in the associations of vowel letters "vowels" with perceived colors for more than 1,000 people (Cuskley 2019). More interestingly, adults can gain synesthesia by training (Bor 2014).

\section{Audio light systems}

The idea of linking sounds with colors is an old idea, and novel ideas raised with the development of music over the past four hundred years. The modern scientific efforts exerted in this field are related to electronic applications such as smart screens and lighting devices in various fields of their uses and for software applications or any other electronic circuit artefact (Lunke2020; Claus2003; New1983). The proposed methods found in the literature depend on dividing the light spectrum into several equal intervals, and each interval is focusing on a specific color. The same method is used for the auditory intervals, (i.e., sound with a specific interval). Then, each sound interval is linked to a light interval. As for the colors, RGB scale or HSB-HSL scale 


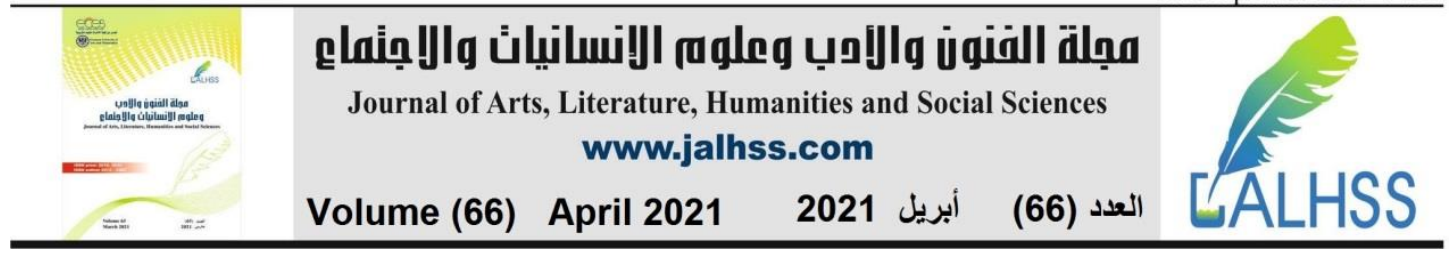

are used by mixing red, green and blue (Claus2003). We also did not find ways to indicate that the sound waves are linked to the visual ones. This may be due to the difficulty of comparing the two waves of different nature, as it will be discussed later.

We may find in the literature a method of drawing the sound as a wave, an oscillation, a mix of different wavelengths, such as drawing a name or phrase in the form of studied vibrations after analyzing the sound signals by means of a microphone and an adequate audio software. But it is devoid of colors, and if they exist, then they are not based on scientific attribution (Soundsnap 2020). This method is often used by music professionals and not by the plastic artist.

From this standpoint, we began our search for a logical scientific solution without neglecting the testimonies and opinions of people who were previously mentioned here above. We began to study the relationship of colors to musical notes and their movements and what they have in common.

\section{Linking sound to colors and HK scale}

Every sound is a mechanical vibration, or a mechanical wave and every wave has a length or a frequency. This frequency is known, for example, for the A4 key on the piano to be $440 \mathrm{~Hz}$. There are many ways to calculate the frequency of each note in the acoustic field from the $440 \mathrm{~Hz}$ value, such as the equal tempered scale, Pythagorean scale, and the pentagonal or hexagonal scale (Isacoff 2001). These scales are used to calculate the tone frequency per octave: A, A\#, B, B\#, C, C\#, D, D\#, E, F, F\#, G, G\#.

Connecting this mechanical wave to light waves or converting this sounds into a light is practically impossible because the nature of the light wave is electromagnetic, not mechanical. It is known, in practice, that any wave of a certain wavelength has harmonics (octaves). For example, the second harmonic for A4 is A5 $=880 \mathrm{~Hz}$, and the third one is A6 $=1760 \mathrm{~Hz}$, etc.

In theory, if we continued to multiply the frequency of A4 to a large number $2^{\mathrm{n}}$ (where $\mathrm{n}$ is an integer), we would have reached a specific frequency or wavelength of light. This wavelength being trapped between ultraviolet and infrared, must be in the visual field. The resulting wavelength allows the definition of a color for the A4 note and for all the other octave's notes to A5.

For each note a specific color graded according to the spectrum of white light (visible spectrum of light). Based on the spectra found in scientific literature (Brand1995), we were able to make a new scale we called "HK scale of visual music". We have not found it published in any previous scientific work (to the extent of our knowledge). 


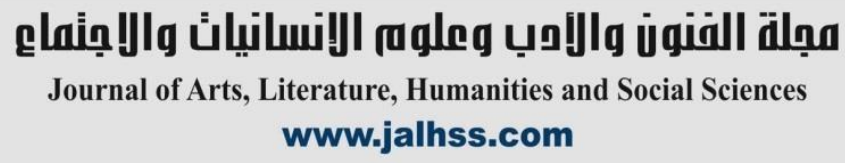

Volume (66) April 2021

2021 بريل

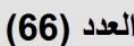

In figure 01, an illustrative oil painting of the positioning of C4 and C5 piano's notes according to the propagation medium of the wave.

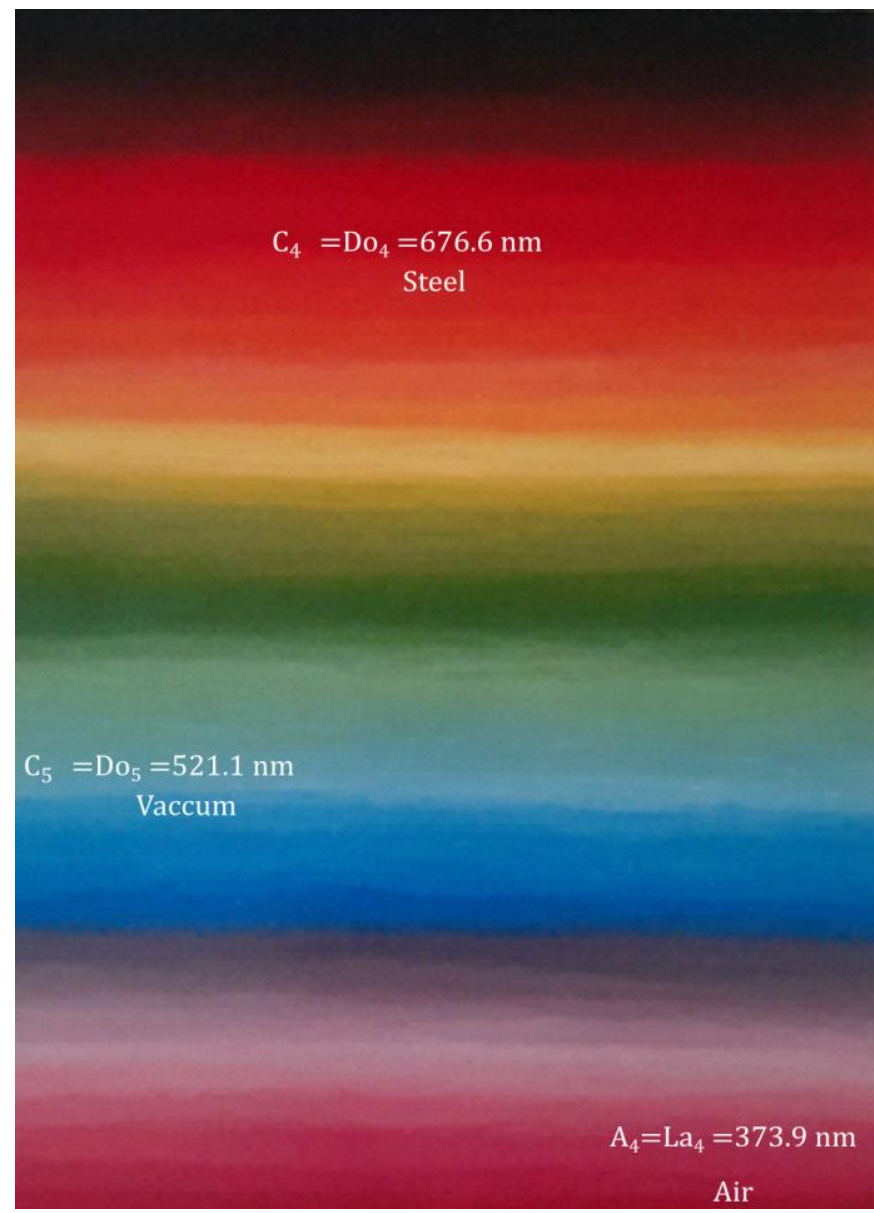

Figure 01: HK scale of visual music. Oil painting on Canvas, fine artist Hadda Zoghbi, 2020. Dimension: A3. C4 is a key equivalent to orange in the air, red in steel. The turquoise color in vacuum is equivalent to $\mathrm{C5}$.

We mentioned earlier that Claus tried to associate notes with colors (Claus2003), and his result is completely different from the HK scale. For example, he defines the wavelength of $\mathrm{A \#}$ to be 650 nanometers and adjusting it with a mixture of red and blue with a ratio of $2 / 1$ and no reference to the medium of propagation of light or sound. While our work directly links A3\# to $705.8 \mathrm{~nm}$ in air and A4\# to $379.4 \mathrm{~nm}$ in steel, and the color wave $548.9 \mathrm{~nm}$ in vacuum connects to A4\#. In addition, Claus or others did not address the issue of the deaf community. 


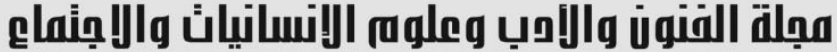 \\ Journal of Arts, Literature, Humanities and Social Sciences www.jalhss.com}

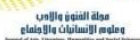

This calculation of the frequencies of light from the sound is depending on the medium in which the mechanical (sound) wave propagates. In air, its velocity is about $350 \mathrm{~m} / \mathrm{s}$, and in metals such as steel the speed is about $5940 \mathrm{~m} / \mathrm{s}$. While the speed of light in vacuum or air is about 300 million $\mathrm{m} / \mathrm{s}$ and this wave does not propagate except in transparent mediums in general, so it is impossible for light to propagate through steel, for example.

We can understand the mechanical or sound wave through the sense of hearing (the frequency of the auditory wave is between $10 \mathrm{~Hz}$ and $20 \mathrm{kHz}$ ) when it travels in the air or by the sense of touch if it is in steel. But the light wave can be understood by sight (the optical wavelength is between 380 and $800 \mathrm{~nm}$, range of human eye sensitivity).

There are a small number of people 1 in 1000 known to have synchronous sensation or Synaesthesia. They can associate sound with color, but it is not certain that these colors represent harmonics, but the proposed scale (HK scale of visual music) provides in a logical and clear manner the relationship between light and sound and does not negate the simultaneous sensation.

Through HK scale of visual music, we can transform every musical clip into a visual painting, and on this basis, we transformed one of Beethoven's piece of music into one visual painting for the listener and a second one for the deaf, respecting the duration of each note. The result is shown in figure 02 . 


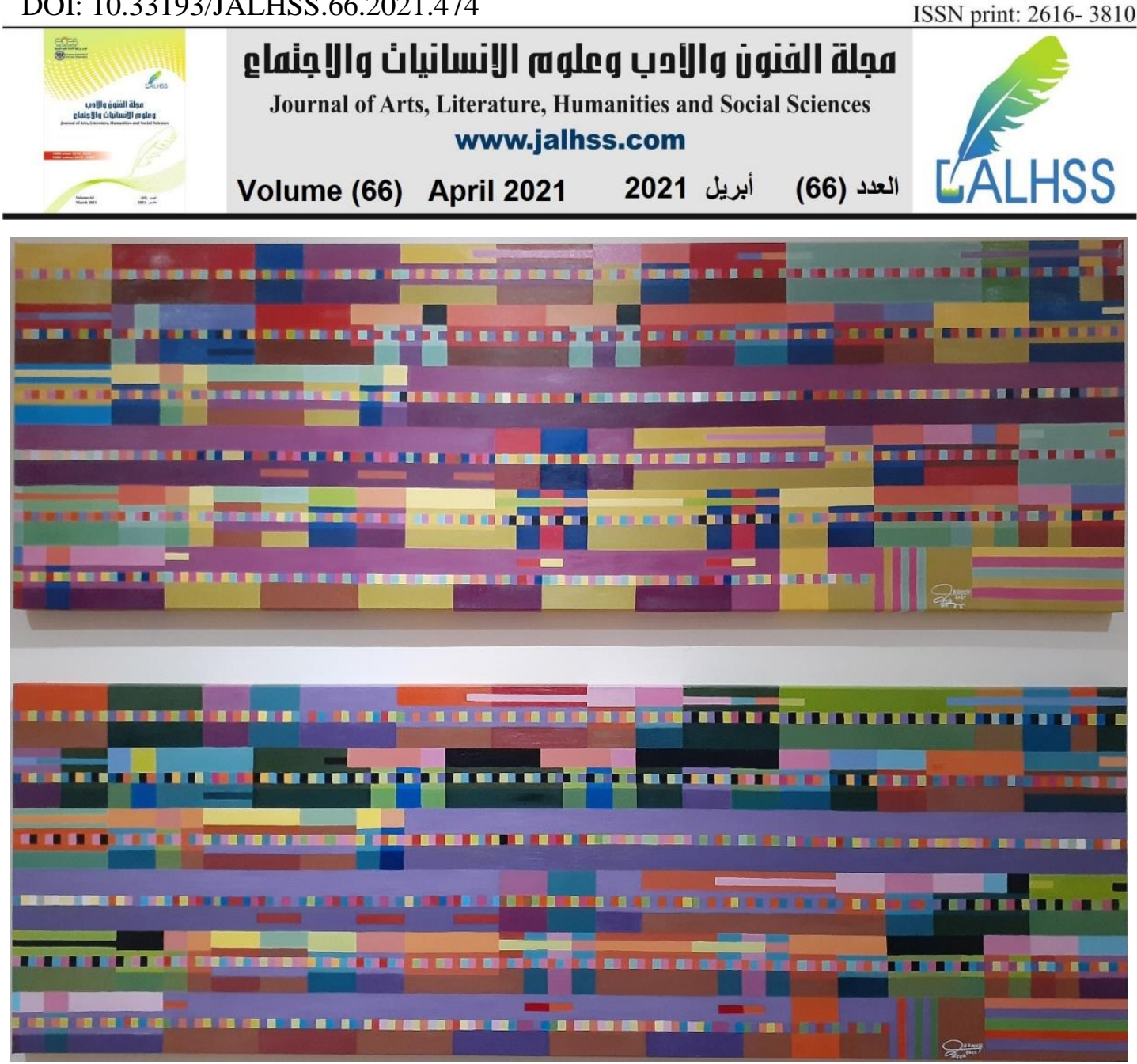

Figure 02: The top image: Beethoven sonata No. 14 (part 01) for the listener, 2020. Oil on Canevas. $180 \mathrm{~cm} \times 60 \mathrm{~cm}$. The Bottom image: Beethoven sonata No. 14 (part 01) for the deaf, 2020 . Oil on Canevas. $180 \mathrm{~cm} \times 60 \mathrm{~cm}$. Fine artist Hadda Zoghbi.

During our drawing of the paintings, we tried to simplify them to a simple form for the musician, so he could look at the painting and play through it, and to achieve that we simplified and clarified the duration of each note (but he must be aware of HK scale). We have also transformed the music of the national anthem of the Kingdom of Bahrain into a painting, part of it for the listener and the other part for the deaf. The result is shown in figure 03 . 


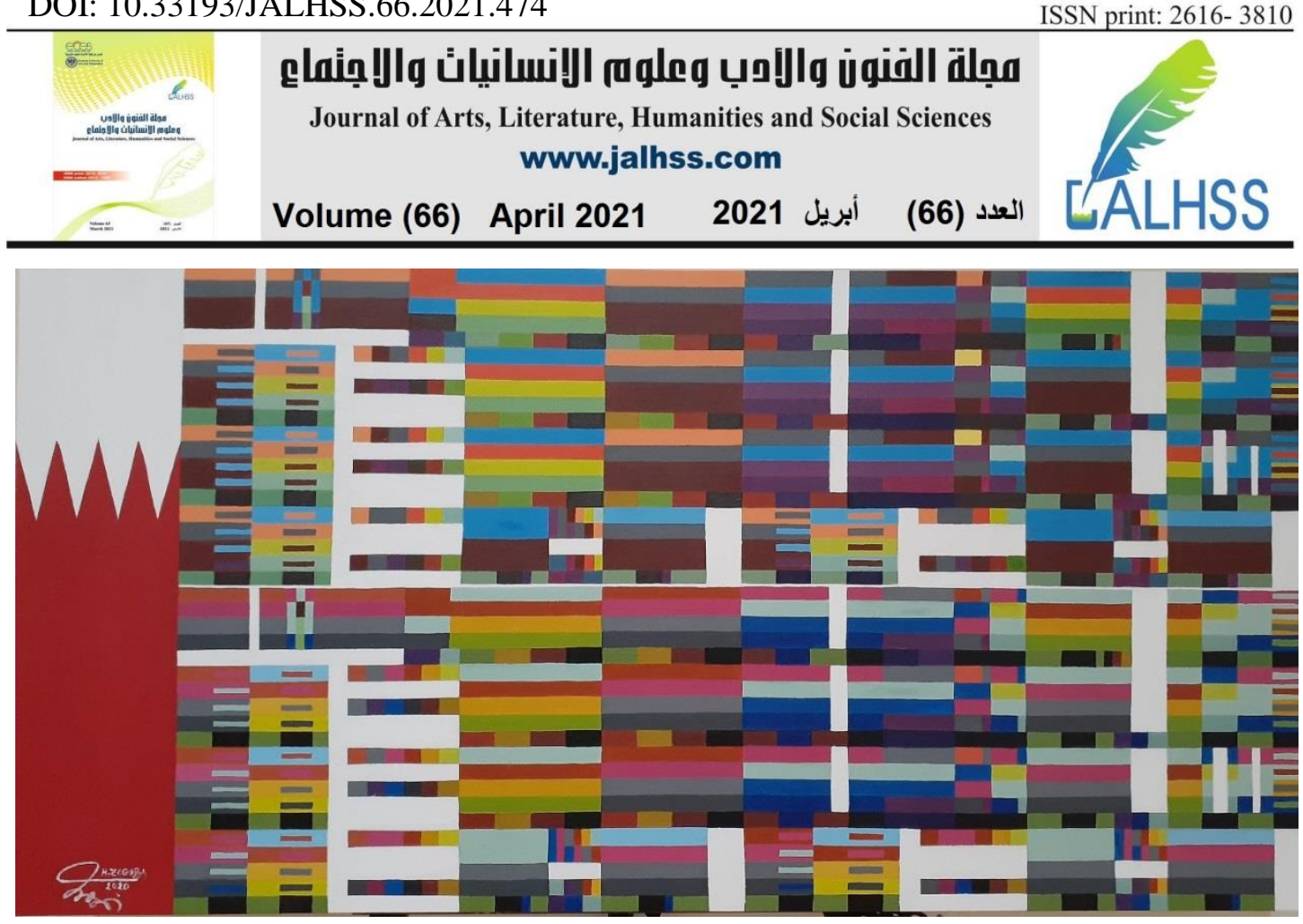

Figure 03: The Bahraini national anthem, 2020 (five wind musical instruments, the upper part for the listener, and the lower part for the deaf). Oil on Canevas.120cm $\times 60 \mathrm{~cm}$. Fine artist Hadda Zoghbi.

For notes with a frequency outside the visual field, we linked them to the colors as follows: Orange (in air) is equivalent to C4, C3 (infrared rays) is dark orange, and C5 (ultraviolet rays) is light orange. The same principle was applied to the rest of the notes.

This way, we have proposed a new approach to abstract and contemporary art, but with a logical and scientific way, mixed with the feelings of the plastic artist, his sympathy and appreciation for this category of disabilities that need support from us so it can break the barrier of isolation with normal people.

This new method does not only open the way for the deaf to understand, enjoy music, or even compose music, but it opens another horizon for the blind to understand colors by listening to music. In other words, it is possible to transform artistic paintings into musical compositions. In addition, psychiatry can use and exploit this new method in psychological treatment. 


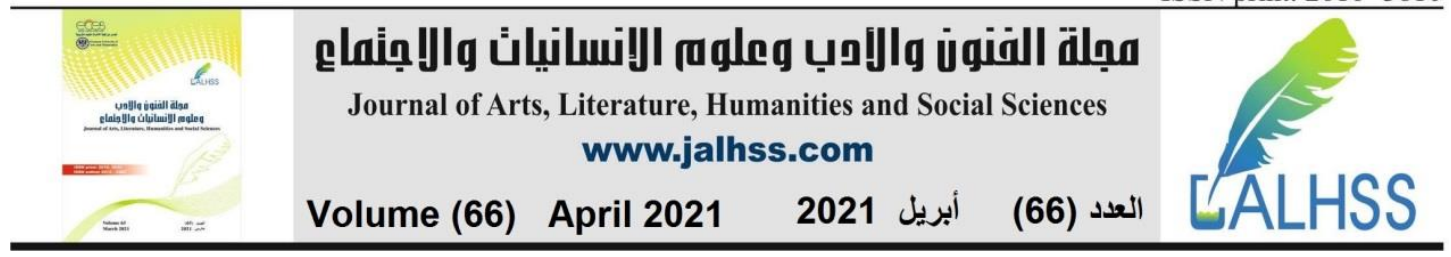

\section{References}

1. (WHO 2020) WHO [online]. Deafness and hearing loss. Available from: https://www.who.int/news-room/fact-sheets/detail/deafness-and-hearing-loss

2. (Mohamad 2011) Mohamad Halil ElRouwili, Deaf and dumb striving to introduce themselves to society, Al-Jazeerah, Saoudi Arabia, Issue, 14098, 05 May 2011.

3. (Aldous 2014) Biography.com Editors [online]. Aldous Huxley Biography. Available from: https://www.biography.com/writer/aldous-huxley

4. (Greenberg 2001) Professor Robert Greenberg, Great Masters: Beethoven: His Life and Music, Edition THE TEACHING COMPANY, 2001

5. (Bredlow 2010) Luis Andrés Bredlow, Aristotle on pre-Platonic theories of sense-perception and knowledge, Filosofi a Unisinos, 11(3):204-224, set/dez 2010

6. (Latest News 2013) Latest News. Classical music, colours and emotions linked, 17 May 2013

7. (Ward 2014) Ossian Ward, How Wassily Kandinsky's synaethesia changed art, The Telegraph, 16 December 2014.

8. (Kandinsky 1911) Wassily Kandinsky. ON THE SPIRITUAL IN ART, 1911, New-York city, HILLA REBAY EDITOR, 1946

9. (Cuskley 2019) Christine Cuskley, Mark Dingemanse, Simon Kirby, Tessa M. van Leeuwen, Cross-modal associations and synesthesia: Categorical perception and structure in vowel-color mappings in a large online sample. Behavior Research Methods, 2019

10. (Lush 2020) Lush P., Botan V., Scott, R.B. et al. Trait phenomenological control predicts experience of mirror synaesthesia and the rubber hand illusion. Nat Commun 11, 4853, 2020

11. (Bor 2014) Bor D., Rothen, N., Schwartzman, D. et al. Adults Can Be Trained to Acquire Synesthetic Experiences. Sci Rep 4, 7089 (2014).

12. (Lunke2020) Lunke K., Meier B., A persistent memory advantage is specific to grapheme-colour synaesthesia. Sci Rep 10, 3484, 2020

13. (Claus 2003) Claus Hvass, Method for conversion of sound signals into light, EP0889746B1, European Patent Office, 2003.

14. (Brand 1995) John C. D. Brand, Tine of light the sources of dispersive spectroscopy, Gordon and Beach Publishers, 1995

15. (Isacoff 2001) Stuart Isacoff, Temperament: How Music Became a Battleground for the Great Minds of Western Civilization, Vintage books, 2001

16. (Soundsnap 2020) Soundsnap [online]. Drawing Sound Effects (1312). Available from: https://www.soundsnap.com/tags/drawing

17. (New 1983) Deborah M. New, US Patent 4627092, 1983 